\title{
Coeffective Basic Cohomologies of $K$-Contact and Sasakian Manifolds
}

\author{
Cristian Ida \& Paul Popescu
}

\begin{abstract}
In this paper, we define coeffective de Rham cohomology for basic forms on a $K$-contact or Sasakian manifold $M$, and we discuss its relation with usual basic cohomology of $M$. When $M$ is of finite type (for instance, it is compact), several inequalities relating some basic coeffective numbers to classical basic Betti numbers of $M$ are obtained. In the case of Sasakian manifolds, we define and study coeffective Dolbeault and Bott-Chern cohomologies for basic forms. Also, in this case, we prove some Hodge decomposition theorems for coeffective basic de Rham cohomology, relating this cohomology with coeffective basic Dolbeault or Bott-Chern cohomology. The notions are introduced in a similar manner with the case of symplectic and Kähler manifolds.
\end{abstract}

\section{Introduction and Preliminary Notions}

\subsection{Introduction}

The coeffective cohomology was introduced by Bouché [6] for symplectic manifolds. More exactly, a symplectic form $\omega$ defines a special subcomplex of the de Rham complex $\left(F^{\bullet}(M), d\right)$ of differential forms on $M$ : it consists of the forms $\varphi$ that are annihilated by $\omega$, that is, $\varphi \wedge \omega=0$. Since $\omega$ is closed, we have in fact a subcomplex of $\left(F^{\bullet}(M), d\right)$ whose cohomology is called coeffective. This cohomology is related with the truncated de Rham cohomology by the class $\omega$. Further significant developments of coeffective cohomologies in many different contexts (symplectic, Kähler, (almost) cosymplectic, (almost) contact, quaternionic manifolds) are given by a series of papers of de Andrés, Fernández, de León, Ibáñez, Mencía, Chinea, Marrero $[2 ; 8 ; 9 ; 14 ; 15 ; 16]$, and other papers by these authors. For Kähler manifolds, both cohomologies (coeffective cohomology and de Rham cohomology truncated by $[\omega])$ are isomorphic for $p \neq n, \operatorname{dim} M=2 n$, though in general they are different for non-Kähler symplectic manifolds [2]. For symplectic manifolds of finite type, the coeffective numbers of the symplectic manifold and several inequalities relating them to the Betti numbers were introduced. Similar results were obtained in the context of almost contact $[9 ; 15]$ and quaternionic

Received October 6, 2014. Revision received June 16, 2015.

The first author is supported by the Sectorial Operational Program Human Resources Development (SOP HRD), financed from the European Social Fund and by the Romanian Government under the Project number POSDRU/159/1.5/S/134378. 
manifolds [16]. Also, a coeffective Dolbeault cohomology for compact Kähler and indefinite Kähler manifolds is studied in [18].

Our aim in this paper is to study the coeffective de Rham, Dolbeault, and BottChern cohomologies for basic forms of (compact) $K$-contact or Sasakian manifolds with respect to the Reeb foliation $\mathcal{F}_{\xi}$ of the fundamental Reeb vector field $\xi$, giving a new contribution concerning basic cohomology of $K$-contact or Sasakian manifolds. Notice that a background about the basic cohomology of $K$-contact and Sasakian manifolds can be found in Chapter VII of [7]. Other developments of basic cohomologies of $K$-contact and Sasakian manifolds in a similar direction as in the recent studies for symplectic manifolds, [27], are given in [19].

The structure of the paper is as follows. In the preliminary subsection, following [7; 23], we briefly recall some elementary definitions about basic forms, basic star operator, basic de Rham Laplacian, and basic de Rham cohomology on $K$-contact manifolds.

We notice that if $\eta$ is the contact form of $M$, then $d \eta$ is basic with respect to the Reeb foliation $\mathcal{F}_{\xi}$. Thus, in Section 2, we begin our study with the coeffective de Rham cohomology for basic forms on a $K$-contact manifold $M$. The main ingredient is given by the isomorphism between the space of basic differential forms on $M$ and the space of differential forms on the orbit space $M_{\xi}$ of $\mathcal{F}_{\xi}$, which is known to be symplectic (or Kählerian in the Sasakian case). Thus, following the classical study of coeffective cohomology of symplectic manifolds (see [14]), we define the coeffective basic de Rham cohomology of $M$ and prove that when $M$ is compact Sasakian,

$$
H^{p}\left(\mathcal{A}_{b}(M)\right) \cong \widetilde{H}_{b}^{p}(M) \quad \forall p \neq n,
$$

where $H^{p}\left(\mathcal{A}_{b}(M)\right)$ denotes the coeffective basic cohomology group of degree $p$ of $M$, and $\widetilde{H}_{b}^{p}(M)$ is the subspace of the basic de Rham cohomology group of $M$ consisting of those classes $a \in H_{b}^{p}(M)$ such that $a \wedge[d \eta]=0$, or in other words, the truncated basic de Rham cohomology group of degree $p$. Notice that for an arbitrary $K$-contact manifold, $H^{p}\left(\mathcal{A}_{b}(M)\right)$ vanishes for every $p \leq n-1$, where $\operatorname{dim} M=2 n+1$. Also, using a technique based on the long exact sequence in cohomology associated with an exact short sequence of complexes, we obtain that the coeffective basic de Rham cohomology groups of a $K$-contact or Sasakian manifold $M$ of finite type have finite dimension. In this case, if we denote by $c_{b}^{p}(M)=\operatorname{dim} H^{p}\left(\mathcal{A}_{b}(M)\right)$, called the coeffective basic numbers of order $p$ of $M$, then they satisfy the following inequalities:

$$
b_{b}^{p}(M)-b_{b}^{p+2}(M) \leq c_{b}^{p}(M) \leq b_{b}^{p}(M)+b_{b}^{p+1}(M) \quad \forall p \geq n+1,
$$

where $b_{b}^{p}(M)=\operatorname{dim} H_{b}^{p}(M)$ is the basic Betti number of order $p$ of $M$.

As a consequence, for a compact Sasakian manifold, we deduce that

$$
c_{b}^{p}(M)=b_{b}^{p}(M)-b_{b}^{p+2}(M) \quad \forall p \geq n+1,
$$

which means that the coeffective basic numbers of a compact Sasakian manifold measure the jumps between the basic Betti numbers. 
In the end of Section 2, using an exact sequence in cohomology (which is the foliation analogue of the Gysin sequence), we prove that the following isomorphism holds:

$$
H^{p}(\mathcal{A}(M)) \cong H^{p-1}\left(\mathcal{A}_{b}(M)\right) \quad \forall p=1, \ldots, 2 n+1,
$$

where $H^{\bullet}(\mathcal{A}(M))$ is the coeffective de Rham cohomology of $M$ considered in [15].

In Section 3, we consider basic complex-valued forms on a Sasakian manifold $M$, and taking into account that $d \eta$ is a basic form of complex type $(1,1)$, in a similar manner with coeffective Dolbeault cohomology of Kähler manifolds (see [18]), we define and study coeffective basic Dolbeault cohomology of a Sasakian manifold. We prove that when $M$ is compact,

$$
H^{r, s}\left(\mathcal{A}_{b}(M)\right) \cong \widetilde{H}_{b}^{r, s}(M) \quad \forall r+s \neq n,
$$

where $H^{r, s}\left(\mathcal{A}_{b}(M)\right)$ denotes the coeffective basic cohomology group of type $(r, s)$ of $M$, and $\widetilde{H}_{b}^{r, s}(M)$ is the truncated basic Dolbeault cohomology group of type $(r, s)$. In the case where $M$ is a compact Sasakian manifold, we prove a Hodge decomposition theorem for coeffective basic de Rham cohomology of $M$, relating this cohomology with coeffective basic Dolbeault cohomology of $M$. Also, several inequalities relating the coeffective basic Hodge numbers to the classical basic Hodge numbers are given similarly as in the de Rham case.

The aim of Section 4 is to construct a coeffective basic Bott-Chern cohomology of a Sasakian manifold $M$. In this sense, we first define basic Bott-Chern and Aeppli cohomology of $M$, and we obtain a Hodge-Bott-Chern decomposition theorem for basic forms of $M$. Next, in a similar manner with the study of coeffective basic de Rham and Dolbeault cohomology of $M$, we define and study a coeffective Bott-Chern cohomology for basic forms on $M$.

The main methods used here are similar and closely related to those used in [6; $9 ; 14 ; 15 ; 16]$.

\subsection{Preliminaries}

Let $(M, F, \xi, \eta, g)$ be a $(2 n+1)$-dimensional almost contact manifold; that is (see $[3 ; 4 ; 7 ; 23]), F$ is a $(1,1)$-tensor field, $\eta$ is a 1 -form, $\xi$ is a vector field, and $g$ is a Riemannian metric on $M$ such that

$$
\begin{aligned}
F^{2} & =-\mathrm{Id}+\eta \otimes \xi, \quad \eta(\xi)=1, \quad \text { and } \\
g(F X, F Y) & =g(X, Y)-\eta(X) \eta(Y)
\end{aligned}
$$

for every $X, Y \in \mathcal{X}(M)$, where Id is the identity transformation. Then we have $F(\xi)=0$ and $\eta(X)=g(X, \xi)$ for all $X \in \mathcal{X}(M)$. The fundamental 2-form $\Phi$ of $M$ is defined by $\Phi(X, Y)=g(X, F Y)$, and the $(2 n+1)$-form $\eta \wedge \Phi^{n}$ is a volume form on $M$. The almost contact metric manifold is said to be: contact if $d \eta=$ $\Phi ; K$-contact if it is contact and $\xi$ is Killing; normal if $[F, F]+2 d \eta \otimes \xi=0$; Sasakian if it is contact and normal. If $M$ is Sasakian manifold, then it is $K$ contact [3]. 
Consider the field $F^{0}(M)=\mathcal{F}(M)$ of smooth real-valued functions defined on $M$. For each $p=1, \ldots, 2 n+1$, denote by $F^{p}(M)$ the module of $p$-forms, by $F(M)=\bigoplus_{p \geq 0} F^{p}(M)$ the exterior algebra of $M$, and by $\langle\cdot, \cdot\rangle$ the natural scalar product on $F(M)$.

Recall that the differential form $\omega$ on $M$ is called basic if it is horizontal (i.e., $\imath_{\xi} \omega=0$, where $\imath_{\xi}$ denotes the interior product with respect to $\xi$ ) and invariant (i.e., $\mathcal{L}_{\xi} \omega=0$, where $\mathcal{L}_{\xi}$ denotes the Lie derivative with respect to $\xi$ ). Denote by $F_{b}^{p}(M)$ the subspace of all basic $p$-forms on the manifold $M$. It is a module over the ring $F_{b}^{0}(M)=\mathcal{F}_{b}(M)$ of basic functions on $M$ (i.e., $\mathcal{L}_{\xi} f=0$ ). Let $F_{b}(M)=\bigoplus_{p \geq 0} F_{b}^{p}(M)$ be the graded algebra of all basic forms on $M$. By the Cartan identity $\mathcal{L}_{\xi}=d_{l \xi}+l_{\xi} d$ we easily obtain that the exterior differential of a basic form is also basic, so we can consider the basic differential $d_{b}=\left.d\right|_{F_{b}^{p}(M)}: F_{b}^{p}(M) \longrightarrow F_{b}^{p+1}(M)$.

Thus, the basic forms constitute a subcomplex $\left(\bigoplus_{p \geq 0} F_{b}^{p}(M), d_{b}\right)$ of the de Rham complex $\left(\bigoplus_{p \geq 0} F^{p}(M), d\right)$. The cohomology of this subcomplex is defined by

$$
\begin{aligned}
H_{b}(M) & =\bigoplus_{p \geq 0} H_{b}^{p}(M), \\
H_{b}^{p}(M) & =\operatorname{ker}\left\{d_{b}: F_{b}^{p}(M) \rightarrow F_{b}^{p+1}(M)\right\} / d_{b}\left(F_{b}^{p-1}(M)\right) .
\end{aligned}
$$

This cohomology plays the role of de Rham cohomology of the orbit space of the $K$-contact manifold $M$, and we call it the basic de Rham cohomology or simply the basic cohomology of $M$. Moreover, the space of basic cohomology $H_{b}^{\bullet}(M)$ is an invariant of the characteristic foliation $\mathcal{F}_{\xi}$ and therefore is an invariant of the $K$-contact structure on the manifold $M$. The relation between the basic cohomology $H_{b}^{\bullet}(M)$ and de Rham cohomology $H^{\bullet}(M)$ of the $K$-contact manifold $M$ is the same as in the general case of a foliation generated by a nonsingular Killing vector field (see, e.g., [26], Thm. 10.13, p. 139). On compact $K$-contact manifolds, the basic cohomology groups enjoy some special properties. In particular, there is a transverse Hodge theory $[7 ; 11 ; 13]$.

Let $\star$ be the usual star operator on $M$. If $\omega \in F_{b}^{p}(M)$, then the $(2 n-p)$-form $l_{\xi} \star \omega$ is basic. Therefore, we can define the basic star operator $\star_{b}: F_{b}^{p}(M) \longrightarrow$ $F_{b}^{2 n-p}(M)$ by

$$
\star_{b} \omega=(-1)^{p} l_{\xi} \star \omega .
$$

Also, the usual scalar product $\langle\cdot, \cdot\rangle$ on $F^{p}(M)$ restricted to basic forms, is denoted by $\langle\cdot, \cdot\rangle_{b}$, and is given by

$$
\langle\omega, \theta\rangle_{b}=\int_{M} \omega \wedge \star_{b} \theta \wedge \eta
$$

for all $\omega, \theta \in F_{b}^{p}(M)$. We denote by the symbol $A^{*}$ the adjoint of the operator $A: F_{b}(M) \rightarrow F_{b}(M)$ with respect to $\langle\cdot, \cdot\rangle_{b}$. As it is well known, the $\langle\cdot, \cdot\rangle_{b}$-adjoint $d_{b}^{*}$ of $d_{b}$ satisfies $d_{b}^{*}=-\star_{b} d_{b} \star_{b}$. 
The basic de Rham Laplacian $\Delta_{b}$ is defined in terms of $d_{b}$ and its adjoint $d_{b}^{*}$ by

$$
\Delta_{b}=d_{b} d_{b}^{*}+d_{b}^{*} d_{b}
$$

The space $\mathcal{H}_{b}^{p}(M)$ of basic harmonic p-forms on $M$ is then defined to be the kernel of $\Delta_{b}: F_{b}^{p}(M) \rightarrow F_{b}^{p}(M)$, and $\mathcal{H}_{b}^{p}(M)=\operatorname{ker} d_{b} \cap \operatorname{ker} d_{b}^{*}$. The transverse Hodge theorem [13] then says that

$$
F_{b}^{p}(M)=\operatorname{Im} d_{b} \oplus \operatorname{Im} d_{b}^{*} \oplus \operatorname{ker} \Delta_{b}
$$

(see also [10]), and each basic cohomology class has an unique harmonic representative, that is,

$$
H_{b}^{p}(M) \cong \mathcal{H}_{b}^{p}(M)
$$

\section{Coeffective de Rham Cohomology for Basic Forms}

Throughout this section, $M$ is a (compact) $K$-contact manifold of dimension $2 n+$ 1 , and sometimes $M$ is Sasakian. We start with a fundamental result, which plays an important role for our purpose.

TheOREM 2.1 [23]. Let $M$ be a $K$-contact manifold of dimension $2 n+1$, and $M_{\xi}$ the orbit space of the Reeb foliation $\mathcal{F}_{\xi}$ defined by $\xi$. If $\pi: M \rightarrow M_{\xi}$ is the natural projection, then $\pi^{*}: F^{p}\left(M_{\xi}\right) \rightarrow F_{b}^{p}(M)$ is an isomorphism.

Proof. Obviously, $\pi^{*}$ is injective. We prove now that for any $\varphi \in F_{b}^{p}(M)$, there exists $\varphi^{\prime} \in F^{p}\left(M_{\xi}\right)$ such that $\varphi=\pi^{*} \varphi^{\prime}$. Since $\varphi$ is horizontal (i.e., $l \xi \varphi=$ $0)$, the values $\varphi\left(X_{1}, \ldots, X_{p}\right)$ can be nonzero only when the tangent vectors $\left\{X_{1}, \ldots, X_{p}\right\} \in T_{x} M$ are orthogonal on $\xi$. But the condition $\mathcal{L}_{\xi} \varphi=0$ shows that $\varphi$ is invariant by the Reeb group $\left\{\Phi_{t}\right\}_{t \in \mathbb{R}}$, that is, $\Phi_{t}^{*} \varphi=\varphi$. It follows that

$$
\varphi\left(\Phi_{t *} X_{1}, \ldots, \Phi_{t *} X_{p}\right)=\varphi\left(X_{1}, \ldots, X_{p}\right),
$$

and so at the point $\pi(x)$, a $p$-form $\varphi_{\pi(x)}^{\prime}$ with the property $\varphi_{x}=\pi^{*} \varphi_{\pi(x)}^{\prime}$ is well defined. But $x$ is arbitrary in $M$, and then the $p$-form $\varphi^{\prime} \in F^{p}\left(M_{\xi}\right)$ with the property $\varphi=\pi^{*} \varphi^{\prime}$ is well defined, which proves that $F_{b}^{p}(M) \subseteq \operatorname{Im} \pi^{*}$.

It remains only to prove that $\operatorname{Im} \pi^{*} \subseteq F_{b}^{p}(M)$. Remark that for any $\varphi^{\prime} \in$ $F^{p}\left(M_{\xi}\right)$, we have $\Phi_{t}^{*} \pi^{*} \varphi^{\prime}=\pi^{*} \varphi^{\prime}, \imath_{\xi} \pi^{*} \varphi^{\prime}=0$; hence, $\mathcal{L}_{\xi} \pi^{*} \varphi^{\prime}=0$, and then $\pi^{*} \varphi^{\prime} \in F_{b}^{p}(M)$.

Also, it is well known that $\left(M_{\xi}, \Omega\right)$ is symplectic with $d \eta=\pi^{*} \Omega$, and when $M$ is Sasakian, $\left(M_{\xi}, \Omega\right)$ is Kählerian. We now have

Lemma 2.1. The operator $L: F_{b}^{p}(M) \rightarrow F_{b}^{p+2}(M)$ defined by $L \varphi=\varphi \wedge d \eta$ is injective for $p \leq n-1$ and surjective for $p \geq n-1$.

Proof. According to [6], we have that the symplectic operator $L^{\prime}: F^{p}\left(M_{\xi}\right) \rightarrow$ $F^{p+2}\left(M_{\xi}\right)$ given by $L^{\prime} \varphi^{\prime}=\varphi^{\prime} \wedge \Omega$ is surjective for $p \geq n-1$ and injective for 
$p \leq n-1$. Now, by Theorem 2.1 , for every $\varphi \in F_{b}^{p}(M)$, there is $\varphi^{\prime} \in F^{p}\left(M_{\xi}\right)$ such that $\varphi=\pi^{*} \varphi^{\prime}$ and

$$
L \varphi=\varphi \wedge d \eta=\pi^{*} \varphi^{\prime} \wedge \pi^{*} \Omega=\pi^{*}\left(\varphi^{\prime} \wedge \Omega\right)=\left(\pi^{*} \circ L^{\prime}\right) \varphi^{\prime} .
$$

Thus, the operator $L$ is also injective for $p \leq n-1$ and surjective for $p \geq n-1$.

Now, as in the case of classical coeffective cohomology (see $[6 ; 9 ; 15]$ ), we consider the subspace $\mathcal{A}_{b}^{p}(M) \subset F_{b}^{p}(M)$ defined by

$$
\mathcal{A}_{b}^{p}(M)=\left\{\varphi \in F_{b}^{p}(M) \mid \varphi \wedge d \eta=0\right\}=\left.\operatorname{ker} L\right|_{F_{b}^{p}(M)} .
$$

A basic form $\varphi \in \mathcal{A}_{b}^{p}(M)$ is said to be a coeffective basic $p$-form on $M$.

Since $d_{b}$ commutes with $L$, we can consider the subcomplex of basic de Rham complex of $M$, namely, $\left(\mathcal{A}_{b}^{\bullet}(M), d_{b}\right)$, called the coeffective basic de Rham complex of $M$. The cohomology groups of this complex are called coeffective basic de Rham cohomology groups of $M$ and are denoted by $H^{p}\left(\mathcal{A}_{b}(M)\right)$.

As a consequence of Lemma 2.1, we get the following:

Proposition 2.1. Let $M$ be a $K$-contact manifold of dimension $2 n+1$. Then $\mathcal{A}_{b}^{p}(M)=\{0\}$ for $p \leq n-1$, and therefore

$$
H^{p}\left(\mathcal{A}_{b}(M)\right)=\{0\} \quad \text { for } p \leq n-1 .
$$

Let us consider the subspace of $H_{b}^{p}(M)$ given by the basic de Rham cohomology classes truncated by the basic de Rham class $[d \eta]$, namely,

$$
\widetilde{H}_{b}^{p}(M)=\left\{a \in H_{b}^{p}(M) \mid a \wedge[d \eta]=0\right\} .
$$

We notice that as in the case of compact cosymplectic manifolds or compact Kähler manifolds (see [9; 18]), we can obtain a relation between the coeffective basic de Rham cohomology of a compact Sasakian manifold $M$ and the basic de Rham cohomology of $M$ truncated by $[d \eta]$ in the following way.

Let us denote by $[\cdot]$ the basic de Rham cohomology classes and by $\{\cdot\}$ the coeffective basic de Rham classes.

Proposition 2.2. For any $K$-contact manifold $M$ of dimension $2 n+1$, the natural mapping

is surjective for $p \geq n$.

$$
\alpha_{p}(\{\varphi\})=[\varphi]
$$

Proof. Let $a \in \widetilde{H}_{b}^{p}(M)$, that is, $a \in H_{b}^{p}(M)$ and $a \wedge[d \eta]=0$ in $H_{b}^{p+2}(M)$. Consider a representative $\varphi$ of $a$ and suppose that $\varphi \notin \mathcal{A}_{b}^{p}(M)$ (notice that if $\varphi \in \mathcal{A}_{b}^{p}(M)$, then $\varphi$ defines a basic cohomology class in $H^{p}\left(\mathcal{A}_{b}(M)\right)$ such that $\left.\alpha_{p}(\{\varphi\})=a\right)$.

Since $a \wedge[d \eta]=0$, there exists $\sigma \in F_{b}^{p+1}(M)$ such that $\varphi \wedge d \eta=d_{b} \sigma$. Then, by Lemma 2.1 there exists $\gamma \in F_{b}^{p-1}(M)$ such that $L \gamma=\sigma$. Thus, $L\left(\varphi-d_{b} \gamma\right)=$ 0 and $d_{b}\left(\varphi-d_{b} \gamma\right)=0$. Hence, $\varphi-d_{b} \gamma$ defines a basic cohomology class in $H^{p}\left(\mathcal{A}_{b}(M)\right)$ such that $\alpha_{p}\left(\left\{\varphi-d_{b} \gamma\right\}\right)=a$. 
We also notice that for compact Sasakian manifolds, we have

$$
\Delta_{b} L=L \Delta_{b}
$$

This follows by a direct calculation using the following known identities from Sasakian geometry $[17 ; 22 ; 23]$ :

$$
\begin{aligned}
\Delta \varphi & =\Delta_{b} \varphi+L \Lambda \varphi+e_{\eta} \Lambda d \varphi-e_{\eta} d \Lambda \varphi, \\
\Delta L \varphi-L \Delta \varphi & =4(n-p-1) L \varphi+4 d e_{\eta} \varphi,
\end{aligned}
$$

and

$$
\left(\Lambda L^{k}-L^{k} \Lambda\right) \varphi=4 k\left[(n-p-k+1) L^{k-1} \varphi+e_{\eta} l \xi L^{k-1} \varphi\right],
$$

where $L^{0} \varphi=\varphi$ and $L^{-1} \varphi=0$. Here $\Delta=d d^{*}+d^{*} d$ is the usual Laplacian on $M, e_{\eta}: F^{p}(M) \rightarrow F^{p}(M)$ is defined by $e_{\eta} \varphi=\eta \wedge \varphi$, and $\Lambda=\star L \star=-\star_{b} L \star b$ is the adjoint of $L$ with respect to $\langle\cdot, \cdot\rangle$ and $\langle\cdot, \cdot\rangle_{b}$, respectively.

Relation (2.4) says that the map $L: \mathcal{H}_{b}^{p}(M) \rightarrow \mathcal{H}_{b}^{p+2}(M)$ is well defined on the space of harmonic basic $p$-forms on $M$. Moreover, by the Bouché result for compact Kähler manifolds (see [6]) and taking into account the Theorem 2.1, we have that

Lemma 2.2. Let $M$ be a compact Sasakian manifold of dimension $2 n+1$. The operator $L: \mathcal{H}_{b}^{p}(M) \rightarrow \mathcal{H}_{b}^{p+2}(M)$ is surjective for $p \geq n-1$.

This also follows directly using Lemma 2.1 and basic Hodge decomposition (1.5). We have the following:

Theorem 2.2. Let $M$ be a compact Sasakian manifold of dimension $2 n+1$. Then

$$
H^{p}\left(\mathcal{A}_{b}(M)\right) \cong \widetilde{H}_{b}^{p}(M) \quad \forall p \neq n .
$$

Proof. We consider two cases.

Case 1: $p \leq n-1$.

From (2.1) we know that $H^{p}\left(\mathcal{A}_{b}(M)\right)=\{0\}$ for $p \leq n-1$. Moreover, from the isomorphism (1.6) we have

$$
\begin{aligned}
\widetilde{H}_{b}^{p}(M) & \cong\left\{\varphi \in \mathcal{H}_{b}^{p}(M) \mid \varphi \wedge d \eta \in d_{b}\left(F_{b}^{p+1}(M)\right)\right\} \\
& \cong\left\{\varphi \in \mathcal{H}_{b}^{p}(M) \mid \varphi \wedge d \eta=0\right\} .
\end{aligned}
$$

Thus, from Lemma 2.1 we conclude that $\widetilde{H}_{b}^{p}(M)=\{0\}$ for $p \leq n-1$. This finishes the proof for $p \leq n-1$.

Case 2: $p \geq n+1$.

We shall see that the mapping $\alpha_{p}$ given by (2.3) is an isomorphism for $p \geq$ $n+1$. By Proposition 2.2 it suffices to show the injection.

Let $a \in H^{p}\left(\mathcal{A}_{b}(M)\right)$ be such that $\alpha_{p}(a)=0$ in $\widetilde{H}_{b}^{p}(M)$ and suppose that $\varphi$ is a representative of $a$. Since $\alpha_{p}(a)=\alpha_{p}(\{\varphi\})=[\varphi]=0$ in $\widetilde{H}_{b}^{p}(M)$, there exists $\psi \in F_{b}^{p-1}(M)$ such that

$$
\varphi=d_{b} \psi
$$


Suppose that $\psi \notin \mathcal{A}_{b}^{p-1}(M)$ (notice that if $\psi \in \mathcal{A}_{b}^{p-1}(M)$, then $a=0$, and we conclude the proof). Since $L$ commutes with $d_{b}$, we have $d_{b}(L \psi)=L\left(d_{b} \psi\right)=$ $L \varphi=0$; therefore, $L \psi$ defines a basic cohomology class $[L \psi] \in H_{b}^{p+1}(M)$. From the isomorphism (1.6) we have

$$
L \psi=h+d_{b} \gamma
$$

for $h \in \mathcal{H}_{b}^{p+1}(M), \gamma \in F_{b}^{p}(M)$. Since $p \geq n+1$ and $h \in \mathcal{H}_{b}^{p+1}(M)$, by Lemma 2.2 there exists $\sigma \in \mathcal{H}_{b}^{p-1}(M)$ such that $L \sigma=h$, and since $p-1 \geq n$, by Lemma 2.1 there exists $\sigma_{1} \in F_{b}^{p-2}(M)$ such that $\gamma=L \sigma_{1}$. Thus,

$$
L\left(\psi-\sigma-d_{b} \sigma_{1}\right)=0 \text { and } d_{b}\left(\psi-\sigma-d_{b} \sigma_{1}\right)=\varphi .
$$

Then $a=\{\varphi\}$ is the basic zero class in $H^{p}\left(\mathcal{A}_{b}(M)\right)$, and this finishes the proof.

Now following an argument similar to that in [15], we relate the coeffective basic de Rham cohomology with the basic de Rham cohomology of $K$-contact manifolds by means of a long exact sequence in basic cohomology.

Let us consider the following short exact sequence for any degree $p$ :

$$
\left.0 \longrightarrow \operatorname{ker} L\right|_{F_{b}^{p}(M)}=\mathcal{A}_{b}^{p}(M) \stackrel{i_{b}}{\longrightarrow} F_{b}^{p}(M) \stackrel{L}{\longrightarrow} \operatorname{Im}_{b}^{p+2} L \longrightarrow 0 .
$$

Since $L$ commutes with $d_{b}$, the sequence (2.7) becomes a short exact sequence of basic differential complexes:

$$
\begin{aligned}
0 \longrightarrow & \left(\left.\operatorname{ker} L\right|_{F_{b}^{p}(M)}, d_{b}\right)=\left(\mathcal{A}_{b}^{p}(M), d_{b}\right) \\
& \stackrel{i_{b}}{\longrightarrow}\left(F_{b}^{p}(M), d_{b}\right) \stackrel{L}{\longrightarrow}\left(\operatorname{Im}_{b}^{p+2} L, d_{b}\right) \longrightarrow 0 .
\end{aligned}
$$

Therefore, we have the associated long exact sequence in cohomology [28]:

$$
\begin{aligned}
\cdots & \longrightarrow H^{p}\left(\mathcal{A}_{b}(M)\right) \stackrel{H\left(i_{b}\right)}{\longrightarrow} H_{b}^{p}(M) \\
& \stackrel{H(L)}{\longrightarrow} H^{p+2}\left(\operatorname{Im}_{b} L\right) \stackrel{\delta_{p+2}^{b}}{\longrightarrow} H^{p+1}\left(\mathcal{A}_{b}(M)\right) \longrightarrow \cdots,
\end{aligned}
$$

where $H\left(i_{b}\right)$ and $H(L)$ are the induced homomorphisms in basic cohomology by $i_{b}$ and $L$, respectively, and $\delta_{p+2}^{b}$ is the connecting homomorphism defined in the following way: if $[\varphi] \in H^{p+2}\left(\operatorname{Im}_{b} L\right)$, then $\delta_{p+2}^{b}[\varphi]=\left[d_{b} \psi\right]$ for $\psi \in F_{b}^{p}(M)$ such that $L \psi=\varphi$.

From Lemma 2.1 it follows that $\operatorname{Im}_{b}^{p+2} L=F_{b}^{p+2}(M)$ for $p \geq n-1$. As a consequence, we have

$$
H^{p+2}\left(\operatorname{Im}_{b} L\right)=H_{b}^{p+2}(M) \quad \forall p \geq n .
$$

Furthermore, the long exact sequence in basic cohomology (2.9) may be expressed as

$$
\begin{aligned}
\cdots & \longrightarrow H^{p}\left(\mathcal{A}_{b}(M)\right) \stackrel{H\left(i_{b}\right)}{\longrightarrow} H_{b}^{p}(M) \\
& \stackrel{H(L)}{\longrightarrow} H_{b}^{p+2}(M) \stackrel{\delta_{p+2}^{b}}{\longrightarrow} H^{p+1}\left(\mathcal{A}_{b}(M)\right) \longrightarrow \cdots
\end{aligned}
$$


for $p \geq n$. Now, we shall decompose the long exact sequence (2.10) into a fiveterm exact sequence:

$$
\begin{aligned}
0 & \rightarrow \operatorname{Im} \delta_{p+1}^{b}=\operatorname{ker} H\left(i_{b}\right) \stackrel{i}{\rightarrow} H^{p}\left(\mathcal{A}_{b}(M)\right) \\
& \stackrel{H\left(i_{b}\right)}{\longrightarrow} H_{b}^{p}(M) \stackrel{H(L)}{\longrightarrow} H_{b}^{p+2}(M) \stackrel{\delta_{p+2}^{b}}{\longrightarrow} \operatorname{Im} \delta_{p+2}^{b} \rightarrow 0 .
\end{aligned}
$$

If $H_{b}^{p}(M)$ are finite-dimensional (for instance if $M$ is compact), then we denote by $b_{b}^{p}(M)=\operatorname{dim} H_{b}^{p}(M)$ the basic $p$ th Betti number of $M$ (see [7]). Since $0 \leq$ $\operatorname{dim}\left(\operatorname{Im} \delta_{p}^{b}\right) \leq b_{b}^{p}(M)$ for $p \geq n+2$, we have the following result.

Proposition 2.3. Let $M$ be a $K$-contact manifold of dimension $2 n+1$ such that $H_{b}^{p}(M)$ are finite-dimensional. Then the coeffective basic de Rham cohomology group $H^{p}\left(\mathcal{A}_{b}(M)\right)$ has finite dimension for $p \geq n+1$.

Thus, for every $p \geq n+1$, we can define the coeffective basic numbers of $M$ by $c_{b}^{p}(M)=\operatorname{dim} H^{p}\left(\mathcal{A}_{b}(M)\right)$. Notice that $c_{b}^{p}(M)=0$ for $p \leq n-1$.

From (2.11) we have

$$
\begin{gathered}
\operatorname{dim}\left(\operatorname{Im} \delta_{p+1}^{b}\right)-\operatorname{dim} H^{p}\left(\mathcal{A}_{b}(M)\right)+\operatorname{dim} H_{b}^{p}(M) \\
-\operatorname{dim} H_{b}^{p+2}(M)+\operatorname{dim}\left(\delta_{p+2}^{b}\right)=0
\end{gathered}
$$

for $p \geq n+1$, from which we deduce

$$
\operatorname{dim}\left(\operatorname{Im} \delta_{p+1}^{b}\right)-c_{b}^{p}(M)+b_{b}^{p}(M)-b_{b}^{p+2}(M)+\operatorname{dim}\left(\operatorname{Im} \delta_{p+2}^{b}\right)=0 .
$$

Now, as a consequence of (2.12), we obtain that the coeffective basic numbers of $M$ are bounded by upper and lower limits depending on the basic Betti numbers of the $K$-contact manifold $M$.

TheOREM 2.3. Let $M$ be a $K$-contact manifold of dimension $2 n+1$ such that $H_{b}^{p}(M)$ are finite-dimensional. Then

$$
b_{b}^{p}(M)-b_{b}^{p+2}(M) \leq c_{b}^{p}(M) \leq b_{b}^{p}(M)+b_{b}^{p+1}(M)
$$

for every $p \geq n+1$.

Since $b_{b}^{2 n}(M)=1$ and $b_{b}^{p}(M)=0$ for every $p \geq 2 n+1$, we obtain the following:

Corollary 2.1. Let $M$ be a $K$-contact manifold of dimension $2 n+1$. Then $c_{b}^{2 n}(M)=1$.

We also have the following:

Theorem 2.4. Let $M$ be a compact Sasakian manifold of dimension $2 n+1$. Then

$$
c_{b}^{p}(M)=b_{b}^{p}(M)-b_{b}^{p+2}(M) \quad \forall p \geq n+1 .
$$

Proof. The proof follows in a similar manner the proof of Theorem 5.1 from [9] or Theorem 4.1 from [14] and consists of computing the connecting mapping $\delta_{p+2}^{b}$. 
Let $a \in H_{b}^{p+2}(M)$. Taking into account the Hodge theory for basic forms on compact Sasakian manifolds (see [7]), we may consider the unique harmonic representative $\varphi$ of the basic de Rham cohomology class $a$.

Then, by Lemma 2.2, there exists a harmonic basic $p$-form $\psi$ such that $L \psi=\varphi$. The theorem follows by the definition of the connecting homomorphism, $\delta_{p+2}^{b} \varphi=\left[d_{b} \psi\right]=0$.

In the end of this section, we give a relation between the coeffective de Rham cohomology $H^{\bullet}(\mathcal{A}(M))$ of a compact $K$-contact manifold $M$ [14] and our basic coeffective de Rham cohomology of $M$.

Recall that if $M$ is compact, then the Lie group of isometries of the metric $g$ is compact, and then the closure of the subgroup $\{\exp (t \xi)\}_{t \in \mathbb{R}}$ is a compact Abelian Lie group, that is, it is isomorphic to a torus $\mathcal{T}$. Denoting by $F_{b}^{\bullet}(M)^{\mathcal{T}}$ the complex of $\mathcal{T}$-invariant forms on $M$, according to Proposition 7.2.1 from [7], the sequence

$$
0 \longrightarrow F_{b}^{\bullet}(M) \stackrel{l}{\longrightarrow} F^{\bullet}(M)^{\mathcal{T}} \stackrel{l_{\xi}}{\longrightarrow} F_{b}^{\bullet-1}(M) \longrightarrow 0
$$

is an exact sequence of complexes, which leads to the following long exact sequence in cohomology:

$$
\cdots \longrightarrow H_{b}^{p}(M) \stackrel{l_{*}}{\longrightarrow} H^{p}(M) \stackrel{j_{p}}{\longrightarrow} H_{b}^{p-1}(M) \stackrel{\delta_{p}}{\longrightarrow} H_{b}^{p+1}(M) \longrightarrow \cdots
$$

where $\delta_{p}$ is the connecting homomorphism given by $\delta_{p}[\varphi]=[L \varphi]=[d \eta] \cup[\varphi]$, and $j_{p}$ is the composition of the map induced by $l_{\xi}$ with the isomorphism $H^{p}\left(F^{\bullet}(M)^{\mathcal{T}}\right) \cong H^{p}(M)$.

Taking into account that $\iota_{\xi} L=L_{\xi}$, we have that

$$
0 \longrightarrow \mathcal{A}_{b}^{\bullet}(M) \stackrel{{ }^{l}}{\longrightarrow} \mathcal{A}^{\bullet}(M)^{\mathcal{T}} \stackrel{l_{\xi}}{\longrightarrow} \mathcal{A}_{b}^{\bullet-1}(M) \longrightarrow 0
$$

is an exact sequence of coeffective complexes, where $\mathcal{A}^{\bullet}(M)$ is the space of coeffective forms on $M$, that is, of $\varphi \in F^{\bullet}(M)$ such that $L \varphi=0$, and $\mathcal{A}^{\bullet}(M)^{\mathcal{T}}$ is the space of coeffective $\mathcal{T}$-invariant forms.

Now, if we consider the long exact sequence in cohomology (2.16) for coeffective forms, then we obtain that the connecting homomorphism $\delta_{p}$ vanishes for every $p$, so we get the short exact sequence in coeffective cohomology,

$$
0 \longrightarrow H^{p}(\mathcal{A}(M)) \stackrel{j_{p}}{\longrightarrow} H^{p-1}\left(\mathcal{A}_{b}(M)\right) \longrightarrow 0
$$

for every $p \geq 1$, which gives the following:

THeOREM 2.5. If $M$ is a compact $K$-contact manifold of dimension $2 n+1$, then

$$
H^{p}(\mathcal{A}(M)) \cong H^{p-1}\left(\mathcal{A}_{b}(M)\right) \quad \forall p=1, \ldots, 2 n+1 .
$$

\section{Coeffective Basic Dolbeault Cohomology}

In this section, we extend our study for basic forms with complex values on a Sasakian manifold $M$ obtaining a coeffective basic Dolbeault cohomology on $M$. In the case where $M$ is a compact Sasakian manifold, we prove a Hodge decomposition theorem for coeffective basic de Rham cohomology of $M$, relating this 
cohomology with basic coeffective Dolbeault cohomology of $M$. The notions are introduced in a similar manner as for Kähler manifolds; see [18].

For our purpose, the complex-valued forms on Sasakian manifolds play an important role. For this reason, we need to recall some notions about Dolbeault basic operators on Sasakian manifolds. Notice that an endomorphism $F$ determines a complex structure on the contact distribution $\mathcal{D}=\operatorname{ker} \eta$ and that on a Sasakian manifold we have $N_{F}(X, Y)=0$ for any $X, Y \in \mathcal{D}$, where $N_{F}$ denotes the Nijenhuis tensor associated to $F$. Then the complexified space of basic $p$-forms admits the decomposition

$$
F_{b}^{p}(M) \otimes_{\mathbb{R}} \mathbb{C}=\bigoplus_{r+s=p} F_{b}^{r, s}(M),
$$

where $F_{b}^{r, s}(M)$ is the space of basic forms of type $(r, s)$, that is, the basic forms that can be nonzero only when they act on $r$ vector fields from $\mathcal{D}^{1,0}$ and on $s$ vector fields from $\mathcal{D}^{0,1}$. Here we have considered the decomposition of the complexified contact distribution, namely, $\mathcal{D} \otimes_{\mathbb{R}} \mathbb{C}=\mathcal{D}^{1,0} \oplus \mathcal{D}^{0,1}$. Then, by applying the classical method used in the case of almost complex manifolds (see, e.g., [20], p. 125-126) a simple calculation proves that

$$
d_{b} F_{b}^{r, s}(M) \subset F_{b}^{r+1, s}(M) \oplus F_{b}^{r, s+1}(M),
$$

and so the basic exterior derivative admits the decomposition $d_{b}=\partial_{b}+\bar{\partial}_{b}$, where

$$
\partial_{b}: F_{b}^{r, s}(M) \rightarrow F_{b}^{r+1, s}(M) ; \quad \bar{\partial}_{b}: F_{b}^{r, s}(M) \rightarrow F_{b}^{r, s+1}(M) .
$$

By $d_{b}^{2}=0$ we deduce

$$
\partial_{b}^{2}=\bar{\partial}_{b}^{2}=\partial_{b} \bar{\partial}_{b}+\bar{\partial}_{b} \partial_{b}=0 .
$$

On the other hand, we have the decomposition $d_{b}^{*} \omega=\partial_{b}^{*} \omega+\bar{\partial}_{b}^{*} \omega$, induced by the decomposition $d_{b}=\partial_{b}+\bar{\partial}_{b}$ of the basic differential and some formulas similar to (3.2), namely,

$$
\partial_{b}^{* 2}=\bar{\partial}_{b}^{* 2}=\partial_{b}^{*} \bar{\partial}_{b}^{*}+\bar{\partial}_{b}^{*} \partial_{b}^{*}=0 .
$$

Notice that the classical Hodge identities from Kähler geometry also hold on a compact Sasakian manifold, as shown in [25]. See also Lemma 7.2.7 from [7] or Lemma 3.4.4 from [11] in a more general case of transversally Kählerian foliations. If we define

$$
\Delta_{b}=d_{b} d_{b}^{*}+d_{b}^{*} d_{b}, \quad \Delta_{\partial_{b}}=\partial_{b} \partial_{b}^{*}+\partial_{b}^{*} \partial_{b}, \quad \Delta_{\bar{\partial}_{b}}=\bar{\partial}_{b} \bar{\partial}_{b}^{*}+\bar{\partial}_{b}^{*} \bar{\partial}_{b},
$$

then we have the following:

Lemma 3.1 ([7]). On a compact Sasakian manifold, we have

$$
\Delta_{b}=\Delta_{\partial_{b}}+\Delta_{\bar{\partial}_{b}}=2 \Delta_{\partial_{b}}=2 \Delta_{\bar{\partial}_{b}} .
$$

Also, the equality $\bar{\partial}_{b}^{2}=0$ induces the differential complex $\left(F_{b}^{r, \bullet}(M), \bar{\partial}_{b}\right)$; its cohomology groups

$$
H_{b}^{r, s}(M)=\operatorname{ker}\left\{F_{b}^{r, s}(M) \stackrel{\bar{\partial}_{b}}{\rightarrow} F_{b}^{r, s+1}(M)\right\} / \bar{\partial}_{b}\left(F_{b}^{r, s-1}(M)\right)
$$


are the analogs of Dolbeault cohomology groups from Kähler geometry (see [20; 21]) and are called the basic Dolbeault cohomology groups of the Sasakian manifold $M$ [7]. In particular, there is a transverse Hodge theory for the operator $\bar{\partial}_{b}$; see $[7 ; 12]$.

Since $d \eta \in F_{b}^{1,1}(M)$, as in the previous subsection, we consider the subspace $\mathcal{A}_{b}^{r, s}(M) \subset F_{b}^{r, s}(M)$ defined by

$$
\mathcal{A}_{b}^{r, s}(M)=\left\{\varphi \in F_{b}^{r, s}(M) \mid \varphi \wedge d \eta=0\right\}=\left.\operatorname{ker} L\right|_{F_{b}^{r, s}(M)} .
$$

A basic form $\varphi \in \mathcal{A}_{b}^{r, s}(M)$ is said to be a coeffective (bigraduate) basic form of bidegree $(r, s)$.

By $\bar{\partial}_{b} d \eta=0$ the operator $L$ commutes with the operator $\bar{\partial}_{b}$. Therefore, we can consider the subcomplex of basic Dolbeault complex of $M$, namely, $\left(\mathcal{A}_{b}^{r, \bullet}, \bar{\partial}_{b}\right)$ for $0 \leq r \leq n$; it is called the coeffective basic Dolbeault complex of $M$. The cohomology groups of this subcomplex are called coeffective basic Dolbeault cohomology groups of $M$ and are denoted by $H^{r, s}\left(\mathcal{A}_{b}(M)\right)$.

Taking into account the decomposition (3.1), we obtain the following version of Lemma 2.1 when $L$ acts on $F_{b}^{r, s}(M)$.

Lemma 3.2. The operator $L: F_{b}^{r, s}(M) \rightarrow F_{b}^{r+1, s+1}(M)$ defined by $L \varphi=\varphi \wedge d \eta$ is injective for $r+s \leq n-1$ and surjective for $r+s \geq n-1$.

As a consequence of Lemma 3.2, we get the following:

Proposition 3.1. Let $M$ be a regular Sasakian manifold of dimension $2 n+1$. Then $\mathcal{A}_{b}^{r, s}(M)=\{0\}$ for $r+s \leq n-1$, and therefore

$$
H^{r, s}\left(\mathcal{A}_{b}(M)\right)=\{0\} \quad \text { for } r+s \leq n-1 .
$$

Let us denote by $[d \eta]_{D}$ the basic Dolbeault class of $d \eta$ in $H_{b}^{1,1}(M)$ and consider the subspace of $H_{b}^{r, s}(M)$ given by the basic Dolbeault cohomology classes truncated by the class $[d \eta]_{D}$, namely,

$$
\widetilde{H}_{b}^{r, s}(M)=\left\{a \in H_{b}^{r, s}(M) \mid a \wedge[d \eta]_{D}=0\right\} .
$$

Next, we define the mapping $\alpha_{r, s}: H^{r, s}\left(\mathcal{A}_{b}(M)\right) \rightarrow \widetilde{H}_{b}^{r, s}(M)$ by

$$
\alpha_{r, s}\left(\{\varphi\}_{D}\right)=[\varphi]_{D},
$$

where $\{\varphi\}_{D}$ denotes the cohomology class of a coeffective basic form $\varphi$ in $H^{r, s}\left(\mathcal{A}_{b}(M)\right)$, and $[\varphi]_{D}$ denotes the cohomology class of a basic form $\varphi$ in $H_{b}^{r, s}(M)$. This mapping allows us to give the following relation between the coeffective basic Dolbeault cohomology groups of the Sasakian manifold $M$ and the subspaces of the basic Dolbeault cohomology groups given by (3.6).

Proposition 3.2. If $M$ is a regular Sasakian manifold of dimension $2 n+1$, then the mapping $\alpha_{r, s}$ defined by (3.7) is surjective for $r+s \geq n$.

Proof. It follows in a similar manner as in the proof of Proposition 2.6 from [18] (for Kähler manifolds) using the same technique as in Proposition 2.2. 
In the following, we relate the coeffective basic Dolbeault cohomology groups and the subspaces of the basic Dolbeault cohomology groups given by (3.6) for compact Sasakian manifolds, and we prove a coeffective version of the basic Hodge decomposition theorem for coeffective basic Dolbeault cohomology.

Now by Lemma 3.1 and taking into account that $\Delta_{\bar{\partial}_{b}}$ preserves the bigraduation of basic forms, we have the following version of Lemma 2.2 when $L$ acts on the space $\mathcal{H}_{b}^{r, s}(M)=\operatorname{ker} \Delta_{\bar{\partial}_{b}}$ of harmonic basic forms of type $(r, s)$.

Lemma 3.3. The operator $L: \mathcal{H}_{b}^{s-1, s-1}(M) \rightarrow \mathcal{H}_{b}^{r, s}(M)$ is surjective for $r+s \geq$ $n+1$.

Theorem 3.1. For a compact Sasakian manifold $M$ of dimension $2 n+1$, we have

$$
H^{r, s}\left(\mathcal{A}_{b}(M)\right) \cong \widetilde{H}_{b}^{r, s}(M),
$$

for $r+s \neq n$.

Proof. It follows in a similar manner as in the proof of Theorem 3.2 from [18] (for compact Kähler manifolds), using the same technique as in Theorem 2.2.

Now, using this result, by similar arguments as in the proof of Theorem 3.3 from [18] (for Kähler manifolds) we will obtain a Hodge decomposition theorem for coeffective basic Dolbeault cohomology of compact Sasakian manifolds.

TheOREM 3.2. If $M$ is a compact Sasakian manifold of dimension $2 n+1$, then we have

(i) $\widetilde{H}^{p}(M) \cong \bigoplus_{r+s}^{p} \widetilde{H}_{b}^{r, s}(M)$;

(ii) $H^{p}\left(\mathcal{A}_{b}(M)\right) \cong \bigoplus_{r+s}^{p} H^{r, s}\left(\mathcal{A}_{b}(M)\right)$ for $r+s \geq n+1$.

Proof. Let $a \in \widetilde{H}_{b}^{p}(M)$, and let $\varphi$ be a representative of $a$. Without loss the generality, we can assume that $\varphi$ is basic harmonic. From (3.1) we have the decomposition

$$
\varphi=\varphi_{p, 0}+\cdots+\varphi_{r, s}+\cdots+\varphi_{0, p},
$$

and taking into account that $\operatorname{ker} \Delta_{b}=\operatorname{ker} \Delta_{\bar{\partial}_{b}}$, since $\Delta_{\bar{\partial}_{b}} \varphi=\Delta_{b} \varphi=0$ and $\Delta_{\bar{\partial}_{b}}$ preserves the bigraduation, we have

$$
\Delta_{\bar{\partial}_{b}} \varphi_{p, 0}=\cdots=\Delta_{\bar{\partial}_{b}} \varphi_{r, s}=\cdots=\Delta_{\bar{\partial}_{b}} \varphi_{0, p}=0 .
$$

Moreover, since $d \eta$ is of bidegree $(1,1)$ basic form and $\varphi \wedge d \eta=0$, we have

$$
\varphi_{p, 0} \wedge d \eta=\cdots=\varphi_{r, s} \wedge d \eta=\cdots=\varphi_{0, p} \wedge d \eta=0 .
$$

Taking into account the Hodge theory for basic forms on Sasakian manifolds (see [7]), we have

$$
\begin{aligned}
\widetilde{H}_{b}^{r, s}(M) & \cong\left\{\varphi \in \mathcal{H}_{b}^{r, s}(M) \mid \varphi \wedge d \eta \in \bar{\partial}_{b}\left(F_{b}^{r+1, s}(M)\right)\right\} \\
& \cong\left\{\varphi \in \mathcal{H}_{b}^{r, s}(M) \mid \varphi \wedge d \eta=0\right\} .
\end{aligned}
$$

Thus, part (i) follows by (3.9). 
Now, from part (i), Theorem 2.2, and Theorem 3.1 we have

$$
H^{p}\left(\mathcal{A}_{b}(M)\right) \cong \widetilde{H}_{b}^{p}(M) \cong \bigoplus_{r+s=p} \widetilde{H}_{b}^{r, s}(M) \cong \bigoplus_{r+s=p} H^{r, s}\left(\mathcal{A}_{b}(M)\right),
$$

and part (ii) follows.

Let us denote $c_{b}^{r, s}(M)=\operatorname{dim} H^{r, s}\left(\mathcal{A}_{b}(M)\right)$.

COROLlary 3.1. For a compact Sasakian manifold of dimension $2 n+1$, we have

$$
c_{b}^{p}(M)=\sum_{r+s=p} c_{b}^{r, s}(M)
$$

for $p \geq n+1$.

REMARK 3.1. Using the same technique as in the previous section, we can relate the coeffective basic Dolbeault cohomology of Sasakian manifolds by means of a long exact sequence in basic cohomology, and we can prove that

$$
h_{b}^{r, s}(M)-h_{b}^{r+1, s+1}(M) \leq c_{b}^{r, s}(M) \leq h_{b}^{r, s}(M)+h_{b}^{r, s+1}(M) \quad \forall r+s \geq n+1,
$$

where $h_{b}^{r, s}(M)=\operatorname{dim} H_{b}^{r, s}(M)$ are the basic Hodge $(r, s)$-numbers of $M$.

Also, when $M$ is compact, we obtain

$$
c_{b}^{r, s}(M)=h_{b}^{r, s}(M)-h_{b}^{r+1, s+1}(M) \quad \forall r+s \geq n+1 .
$$

\section{Coeffective Basic Bott-Chern Cohomology}

In this section, we first define the basic Bott-Chern and Aeppli cohomology of a Sasakian manifold $M$, and we obtain a Hodge-Bott-Chern decomposition theorem for basic forms of $M$. Next, in similar manner with the study of coeffective basic de Rham and Dolbeault cohomology of $M$, we define and study a coeffective Bott-Chern cohomology for basic forms on $M$.

\subsection{Hodge-Bott-Chern Decomposition for Basic Forms}

In the first part of this subsection, we define the basic Bott-Chern and Aeppli cohomology groups of $M$. In the second part, we define a basic Bott-Chern Laplacian and obtain a Hodge-Bott-Chern-type decomposition theorem for basic forms on $M$.

DEFinition 4.1. The differential complex

$$
\begin{aligned}
\cdots & \longrightarrow F_{b}^{r-1, s-1}(M) \stackrel{\partial_{b} \bar{\partial}_{b}}{\longrightarrow} F_{b}^{r, s}(M) \\
& \stackrel{\partial_{b} \oplus \bar{\partial}_{b}}{\longrightarrow} F_{b}^{r+1, s}(M) \oplus F_{b}^{r, s+1}(M) \longrightarrow \cdots
\end{aligned}
$$


is called the basic Bott-Chern complex of $M$, and the basic Bott-Chern cohomology groups of $M$ of bidegree $(r, s)$ are given by

$$
\begin{aligned}
& H_{b, \mathrm{BC}}^{r, s}(M) \\
& \quad=\frac{\operatorname{ker}\left\{\partial_{b}: F_{b}^{r, s}(M) \rightarrow F_{b}^{r+1, s}(M)\right\} \cap \operatorname{ker}\left\{\bar{\partial}_{b}: F_{b}^{r, s}(M) \rightarrow F_{b}^{r, s+1}(M)\right\}}{\operatorname{Im}\left\{\partial_{b} \bar{\partial}_{b}: F_{b}^{r-1, s-1}(M) \rightarrow F_{b}^{r, s}(M)\right\}} .
\end{aligned}
$$

Next, as in the classical case (see $[1 ; 5 ; 24]$ ), we consider the dual of the basic Bott-Chern cohomology groups given by

$$
H_{b, A}^{r, s}(M)=\frac{\operatorname{ker}\left\{\partial_{b} \bar{\partial}_{b}: F_{b}^{r, s}(M) \rightarrow F_{b}^{r+1, s+1}(M)\right\}}{\operatorname{Im}\left\{\partial_{b}: F_{b}^{r-1, s}(M) \rightarrow F_{b}^{r, s}(M)\right\}+\operatorname{Im}\left\{\bar{\partial}_{b}: F_{b}^{r, s-1}(M) \rightarrow F_{b}^{r, s}(M)\right\}}
$$

and called the basic Aeppli cohomology groups of bidegree $(r, s)$ of $M$.

Proposition 4.1. The exterior product induces a bilinear map

$$
\wedge: H_{b, \mathrm{BC}}^{p, q}(M) \times H_{b, A}^{r, s}(M) \rightarrow H_{b, A}^{p+r, q+s}(M) .
$$

Proof. Let $\varphi, \psi \in F_{b}^{r, s}(M)$. If $\varphi$ is $d_{b}$-closed and $\psi$ is $\partial_{b} \bar{\partial}_{b}$-closed, then $\varphi \wedge \psi$ is $\partial_{b} \bar{\partial}_{b}$-closed. Also, if $\varphi$ is $d_{b}$-closed and $\psi$ is $d_{b}$-exact, then $\varphi \wedge \psi$ is $d_{b}$-exact, and if $\varphi$ is $\partial_{b} \bar{\partial}_{b}$-exact and $\psi$ is $\partial_{b} \bar{\partial}_{b}$-closed, then $\varphi \wedge \psi$ is $d_{b}$-exact.

For the last assertion, we have

$$
\varphi \wedge \psi=\partial_{b} \bar{\partial}_{b} \theta \wedge \psi=\frac{1}{2} d_{b}\left[\left(\bar{\partial}_{b}-\partial_{b}\right) \theta \wedge \psi+(-1)^{r+s} \theta \wedge\left(\partial_{b}-\bar{\partial}_{b}\right) \psi\right] .
$$

In particular,

$$
H_{b, \mathrm{BC}}^{r, s}(M) \times H_{b, A}^{n-r, n-s}(M) \rightarrow H_{b, A}^{n, n}(M)=H_{b}^{2 n}(M) \cong \mathbb{R} .
$$

In the following, we define the Bott-Chern Laplacian for basic forms of type $(r, s)$ by

$$
\Delta_{\mathrm{BC}}^{b}=\partial_{b} \bar{\partial}_{b}\left(\partial_{b} \bar{\partial}_{b}\right)^{*}+\partial_{b}^{*} \partial_{b}+\bar{\partial}_{b}^{*} \bar{\partial}_{b}
$$

This operator is self-adjoint, that is, $\left\langle\Delta_{\mathrm{BC}}^{b} \varphi, \psi\right\rangle_{b}=\left\langle\varphi, \Delta_{\mathrm{BC}}^{b} \psi\right\rangle_{b}$. Also, for a basic form $\varphi \in F_{b}^{r, s}(M)$, we have

$$
\begin{aligned}
\left\langle\Delta_{\mathrm{BC}}^{b} \varphi, \varphi\right\rangle_{b} & =\left\langle\partial_{b} \bar{\partial}_{b}\left(\partial_{b} \bar{\partial}_{b}\right)^{*} \varphi+\partial_{b}^{*} \partial_{b} \varphi+\bar{\partial}_{b}^{*} \bar{\partial}_{b} \varphi, \varphi\right\rangle_{b} \\
& =\left\langle\left(\partial_{b} \bar{\partial}_{b}\right)^{*} \varphi,\left(\partial_{b} \bar{\partial}_{b}\right)^{*} \varphi\right\rangle_{b}+\left\langle\partial_{b} \varphi, \partial_{b} \varphi\right\rangle_{b}+\left\langle\bar{\partial}_{b} \varphi, \bar{\partial}_{b} \varphi\right\rangle_{b} \\
& =\left\|\left(\partial_{b} \bar{\partial}_{b}\right)^{*} \varphi\right\|^{2}+\left\|\partial_{b} \varphi\right\|^{2}+\left\|\bar{\partial}_{b} \varphi\right\|^{2},
\end{aligned}
$$

where $\|\varphi\|^{2}=\langle\varphi, \varphi\rangle_{b}$. Thus, we obtain the following:

Proposition 4.2. $\Delta_{\mathrm{BC}}^{b} \varphi=0$ if and only if $\left(\partial_{b} \bar{\partial}_{b}\right)^{*} \varphi=\partial_{b} \varphi=\bar{\partial}_{b} \varphi=0$.

We denote by $\mathcal{H}_{b, \mathrm{BC}}^{r, s}(M)$ the space of $\Delta_{\mathrm{BC}}^{b}$-harmonic basic forms of type $(r, s)$ on $M$.

Following the ideas from [27], we now show that $H_{b, \mathrm{BC}}^{*, *}(M)$ is finitedimensional by analyzing the space of its harmonic basic forms. First, let us 
consider a related fourth-order elliptic differential operator (see [12] for general transversally Hermitian foliations), namely,

$$
\widetilde{\Delta}_{\mathrm{BC}}^{b}=\partial_{b} \bar{\partial}_{b} \bar{\partial}_{b}^{*} \partial_{b}^{*}+\bar{\partial}_{b}^{*} \partial_{b}^{*} \partial_{b} \bar{\partial}_{b}+\bar{\partial}_{b}^{*} \partial_{b} \partial_{b}^{*} \bar{\partial}_{b}+\partial_{b}^{*} \bar{\partial}_{b} \bar{\partial}_{b}^{*} \partial_{b}+\bar{\partial}_{b}^{*} \bar{\partial}_{b}+\partial_{b}^{*} \partial_{b}
$$

This operator has the same kernel as $\Delta_{\mathrm{BC}}^{b}$. Indeed,

$$
\begin{aligned}
0 & =\left\langle\varphi, \widetilde{\Delta}_{\mathrm{BC}}^{b} \varphi\right\rangle_{b} \\
& =\left\|\partial_{b} \varphi\right\|^{2}+\left\|\bar{\partial}_{b} \varphi\right\|^{2}+\left\|\left(\partial_{b} \bar{\partial}_{b}\right)^{*} \varphi\right\|^{2}+\left\|\partial_{b} \bar{\partial}_{b} \varphi\right\|^{2}+\left\|\partial_{b}^{*} \bar{\partial}_{b} \varphi\right\|^{2}+\left\|\bar{\partial}_{b}^{*} \partial_{b} \varphi\right\|^{2},
\end{aligned}
$$

and the three additional terms clearly do not give any additional conditions and are automatically zero by the requirement $\partial_{b} \varphi=\bar{\partial}_{b} \varphi=0$. Essentially, the presence of the second-order differential terms ensures that the spaces ker $\Delta_{\mathrm{BC}}^{b}$ and ker $\widetilde{\Delta}_{\mathrm{BC}}^{b}$ coincide. Using the classical Hodge identities for Sasakian manifolds (see Lemma 7.2.7 from [7]) in relation (4.4), we also obtain the following:

Proposition 4.3. If $M$ is a compact Sasakian manifold of dimension $2 n+1$, then

$$
\widetilde{\Delta}_{\mathrm{BC}}^{b}=\Delta_{\bar{\partial}_{b}} \Delta_{\bar{\partial}_{b}}+\partial_{b}^{*} \partial_{b}+\bar{\partial}_{b}^{*} \bar{\partial}_{b}
$$

Moreover, the harmonic spaces $\mathcal{H}_{b}^{r+s}(M) \cap F_{b}^{r, s}(M), \mathcal{H}_{b}^{r, s}(M)$, and $\mathcal{H}_{b, \mathrm{BC}}^{r, s}(M)$ coincide, and also $d \eta$ is harmonic basic $(1,1)$-form with respect to every Laplacian $\Delta_{b}, \Delta_{\bar{\partial}_{b}}$, and $\Delta_{\mathrm{BC}}^{b}$, respectively.

We have now the following:

TheOREM 4.1. Let $M$ be a compact Sasakian manifold of dimension $2 n+1$. Then

(i) $\operatorname{dim} \mathcal{H}_{b, \mathrm{BC}}^{r, s}(M)<\infty$;

(ii) there is an orthogonal decomposition

$$
F_{b}^{r, s}(M)=\mathcal{H}_{b, \mathrm{BC}}^{r, s}(M) \oplus \operatorname{Im}\left(\partial_{b} \bar{\partial}_{b}\right) \oplus\left(\operatorname{Im} \partial_{b}^{*}+\operatorname{Im} \bar{\partial}_{b}^{*}\right) ;
$$

(iii) there are the canonical isomorphisms

$$
\mathcal{H}_{b, \mathrm{BC}}^{r, s}(M) \cong H_{b, \mathrm{BC}}^{r, s}(M) \cong H_{b}^{r, s}(M) .
$$

Proof. (i) Because only the highest order differential need to be kept for computing the principal symbol of a Laplace operator, by the calculations of $\widetilde{\Delta}_{\mathrm{BC}}^{b}$ from Proposition 4.3 it follows that the principal symbol of $\widetilde{\Delta}_{\mathrm{BC}}^{b}$ is equal to that of the square of the operator $\Delta_{\bar{\partial}_{b}}$, and so it is positive. Thus, $\widetilde{\Delta}_{\mathrm{BC}}^{b}$ is elliptic, and hence its kernel $\mathcal{H}_{b, \mathrm{BC}}^{r, s}(M)$ is finite-dimensional.

With $\widetilde{\Delta}_{\mathrm{BC}}^{b}$ elliptic, assertion (ii) then follows directly by applying elliptic theory. For (iii), using the decomposition of (ii), we have

$$
\operatorname{ker}\left(\partial_{b}+\bar{\partial}_{b}\right)=\mathcal{H}_{b, \mathrm{BC}}^{r, s}(M) \oplus \operatorname{Im}\left(\partial_{b} \bar{\partial}_{b}\right)
$$


This must be so since for a form $\varphi \in F_{b}^{r, s}(M)$ given by $\varphi=\psi+\partial_{b} \bar{\partial}_{b} \theta+\partial_{b}^{*} \theta_{1}+$ $\bar{\partial}_{b}^{*} \theta_{2}$, where $\psi \in \mathcal{H}_{b, \mathrm{BC}}^{r, s}(M)$, we have $\partial_{b} \varphi=\bar{\partial}_{b} \varphi=0$ if and only if

$$
\begin{aligned}
0 & =\left\langle\theta_{1}, \partial_{b}\left(\partial_{b}^{*} \theta_{1}+\bar{\partial}_{b}^{*} \theta_{2}\right)\right\rangle_{b}+\left\langle\theta_{2}, \bar{\partial}_{b}\left(\partial_{b}^{*} \theta_{1}+\bar{\partial}_{b}^{*} \theta_{2}\right)\right\rangle_{b} \\
& =\left\langle\partial_{b}^{*} \theta_{1}+\bar{\partial}_{b}^{*} \theta_{2}, \partial_{b}^{*} \theta_{1}+\bar{\partial}_{b}^{*} \theta_{2}\right\rangle_{b} \\
& =\left\|\partial_{b}^{*} \theta_{1}+\bar{\partial}_{b}^{*} \theta_{2}\right\|^{2},
\end{aligned}
$$

which implies $\partial_{b}^{*} \theta_{1}+\bar{\partial}_{b}^{*} \theta_{2}=0$, that is, desired decomposition from (4.6). Thus, every cohomology class of $H_{b, \mathrm{BC}}^{\bullet, \bullet}(M)$ contains a unique harmonic representative, and $\mathcal{H}_{b, \mathrm{BC}}^{r, s}(M) \cong H_{b, \mathrm{BC}}^{r, s}(M)$, that is, the first isomorphism of (iii). Since $\operatorname{ker} \widetilde{\Delta}_{\mathrm{BC}}^{b}=\operatorname{ker} \Delta_{\bar{\partial}_{b}}$, the second isomorphism of (iii) follows by $H_{b}^{r, s}(M) \cong$ $\mathcal{H}_{b}^{r, s}(M)$ and $\mathcal{H}_{b}^{r, s}(M)=\mathcal{H}_{b, \mathrm{BC}}^{r, s}(M)$.

Corollary 4.1. If $M$ is a compact Sasakian manifold of dimension $2 n+1$, then $H_{b, \mathrm{BC}}^{r, s}(M)$ is finite-dimensional.

Now, let us define the Aeppli Laplacian for basic forms of type $(r, s)$ on $M$ by

$$
\Delta_{A}^{b}=\partial_{b} \partial_{b}^{*}+\bar{\partial}_{b} \bar{\partial}_{b}^{*}+\left(\partial_{b} \bar{\partial}_{b}\right)^{*} \partial_{b} \bar{\partial}_{b},
$$

which is not elliptic, but if we change it by

$$
\widetilde{\Delta}_{A}^{b}=\partial_{b} \partial_{b}^{*}+\bar{\partial}_{b} \bar{\partial}_{b}^{*}+\bar{\partial}_{b}^{*} \partial_{b}^{*} \partial_{b} \bar{\partial}_{b}+\partial_{b} \bar{\partial}_{b} \bar{\partial}_{b}^{*} \partial_{b}^{*}+\partial_{b} \bar{\partial}_{b}^{*} \bar{\partial}_{b} \partial_{b}^{*}+\bar{\partial}_{b} \partial_{b}^{*} \partial_{b} \bar{\partial}_{b}^{*},
$$

this is elliptic.

Now, if we denote $\mathcal{H}_{b, A}^{r, s}(M)=\operatorname{ker} \widetilde{\Delta}_{A}^{b} \cap F_{b}^{r, s}(M)$, then by applying elliptic theory arguments, similar to Theorem 4.1 , we have the following:

THeOREM 4.2. Let $M$ be a compact Sasakian manifold of dimension $2 n+1$. Then

(i) $\operatorname{dim} \mathcal{H}_{b, A}^{r, s}(M)<\infty$;

(ii) there is an orthogonal decomposition

$$
F_{b}^{r, s}(M)=\mathcal{H}_{b, A}^{r, s}(M) \oplus\left(\operatorname{Im} \partial_{b}+\operatorname{Im} \bar{\partial}_{b}\right) \oplus \operatorname{Im}\left(\bar{\partial}_{b}^{*} \partial_{b}^{*}\right) ;
$$

(iii) there is a canonical isomorphism

$$
\mathcal{H}_{b, A}^{r, s}(M) \cong H_{b, A}^{r, s}(M) .
$$

Corollary 4.2. If $M$ is a compact Sasakian manifold, then $H_{b, A}^{r, s}(M)$ is finitedimensional.

Finally, let us remark that $\star_{b}$ gives an isomorphism $H_{b, \mathrm{BC}}^{r, s}(M) \approx H_{b, A}^{n-r, n-s}(M)$.

\subsection{Coeffective Bott-Chern Cohomology for Basic Forms}

In this subsection, we define and study a coeffective Bott-Chern cohomology for basic forms on Sasakian manifolds. 
Since the operator $L$ commutes with both operators $\partial_{b}$ and $\bar{\partial}_{b}$, we can consider the subcomplex of Bott-Chern complex of $M$

$$
\begin{aligned}
\cdots & \longrightarrow \mathcal{A}_{b}^{r-1, s-1}(M) \stackrel{\partial_{b} \bar{\partial}_{b}}{\longrightarrow} \mathcal{A}_{b}^{r, s}(M) \\
& \stackrel{\partial_{b} \oplus \bar{\partial}_{b}}{\longrightarrow} \mathcal{A}_{b}^{r+1, s}(M) \oplus \mathcal{A}_{b}^{r, s+1}(M) \longrightarrow \cdots
\end{aligned}
$$

for $1 \leq r, s \leq n$, called the coeffective basic Bott-Chern complex of $M$. The cohomology groups of the complex (4.10) are called coeffective basic Bott-Chern cohomology groups of $M$ and are denoted by $H_{\mathrm{BC}}^{r, s}\left(\mathcal{A}_{b}(M)\right)$.

By Lemma 3.2 we get the following:

Proposition 4.4. Let $M$ be a regular Sasakian manifold of dimension $2 n+1$. Then

$$
H_{\mathrm{BC}}^{r, s}\left(\mathcal{A}_{b}(M)\right)=\{0\} \quad \text { for } r+s \leq n-1 .
$$

Since $\partial_{b} d \eta=\bar{\partial}_{b} d \eta=0$, we have that $[d \eta]_{\mathrm{BC}} \in H_{\mathrm{BC}}^{1,1}(M)$, and we consider the subspace of $H_{b, \mathrm{BC}}^{r, s}(M)$ given by the basic Bott-Chern cohomology classes truncated by the basic Bott-Chern class $[d \eta]_{\mathrm{BC}}$, namely,

$$
\widetilde{H}_{b, \mathrm{BC}}^{r, s}(M)=\left\{a \in H_{b, \mathrm{BC}}^{r, s}(M) \mid a \wedge[d \eta]_{\mathrm{BC}}=0\right\} .
$$

Next, we define the mapping $\alpha_{r, s}: H_{\mathrm{BC}}^{r, s}\left(\mathcal{A}_{b}(M)\right) \rightarrow \widetilde{H}_{b, \mathrm{BC}}^{r, s}(M)$ by

$$
\alpha_{r, s}\left(\{\varphi\}_{\mathrm{BC}}\right)=[\varphi]_{\mathrm{BC}},
$$

where $\{\varphi\}_{\mathrm{BC}}$ denotes the cohomology class of a coeffective basic form $\varphi$ in $H_{\mathrm{BC}}^{r, s}\left(\mathcal{A}_{b}(M)\right)$, and $[\varphi]_{\mathrm{BC}}$ denotes the basic cohomology class of a basic form $\varphi$ in $H_{b, \mathrm{BC}}^{r, s}(M)$. This mapping allows us to give a relation between the coeffective basic Bott-Chern cohomology groups of the Sasakian manifold $M$ and the subspaces of the basic Bott-Chern cohomology groups given by (4.12), just in the case of coeffective basic de Rham and Dolbeault cohomology of $M$. In the following, our aim is to find a link between the coeffective basic Bott-Chern cohomology groups and the subspaces of the basic Bott-Chern cohomology groups given by (4.12) for compact Sasakian manifolds and to prove a coeffective version of the basic Hodge decomposition theorem for the basic Bott-Chern cohomology.

Proposition 4.5. If $M$ is a regular Sasakian manifold of dimension $2 n+1$, then the mapping $\alpha_{r, s}$ defined by (4.13) is surjective for $r+s \geq n$.

Proof. Let $a \in \widetilde{H}_{b, \mathrm{BC}}^{r, s}(M)$, that is, $a \in H_{b, \mathrm{BC}}^{r, s}(M)$ and $a \wedge[d \eta]_{\mathrm{BC}}=0$ in $H_{b, \mathrm{BC}}^{r+1, s+1}(M)$. Consider a representative $\varphi$ of $a$ and suppose that $\varphi \notin \mathcal{A}_{b}^{r, s}(M)$ (notice that if $\varphi \in \mathcal{A}_{b}^{r, s}(M)$, then $\varphi$ defines a basic cohomology class in $H_{\mathrm{BC}}^{r, s}\left(\mathcal{A}_{b}(M)\right)$ such that $\left.\alpha_{r, s}\left(\{\varphi\}_{\mathrm{BC}}\right)=a\right)$.

Since $a \wedge[d \eta]_{\mathrm{BC}}=0$, there exists $\sigma \in F_{b}^{r, s}(M)$ such that $\varphi \wedge d \eta=\partial_{b} \bar{\partial}_{b} \sigma$. Then, by Lemma 3.2 there exists $\gamma \in F_{b}^{r-1, s-1}(M)$ such that $L \gamma=\sigma$. Thus, 
$L\left(\varphi-\partial_{b} \bar{\partial}_{b} \gamma\right)=0$ and $\partial_{b}\left(\varphi-\partial_{b} \bar{\partial}_{b} \gamma\right)=\bar{\partial}_{b}\left(\varphi-\partial_{b} \bar{\partial}_{b} \gamma\right)=0$. Hence, $\varphi-$ $\partial_{b} \bar{\partial}_{b} \gamma$ defines a basic cohomology class in $H_{\mathrm{BC}}^{r, s}\left(\mathcal{A}_{b}(M)\right)$ such that $\alpha_{r, s}(\{\varphi-$ $\left.\left.\partial_{b} \bar{\partial}_{b} \gamma\right\}_{\mathrm{BC}}\right)=a$.

Now, taking into account relation (2.4), the classical Hodge identities for Sasakian manifolds, and Proposition 4.3, we have

$$
\widetilde{\Delta}_{\mathrm{BC}}^{b} L-L \widetilde{\Delta}_{\mathrm{BC}}^{b}=-2 i \partial_{b} \bar{\partial}_{b},
$$

and so, if $\varphi \in \mathcal{H}_{b, \mathrm{BC}}^{r, s}(M)$, then $L \varphi \in \mathcal{H}_{b, \mathrm{BC}}^{r+1, s+1}(M)$.

THEOREM 4.3. For a compact Sasakian manifold $M$ of dimension $2 n+1$, we have

$$
H_{\mathrm{BC}}^{r, s}\left(\mathcal{A}_{b}(M)\right) \cong \widetilde{H}_{b, \mathrm{BC}}^{r, s}(M)
$$

for $r+s \notin\{n, n+1\}$.

Proof. Using an argument similar to that used in [18], we consider two cases using the same technique as in Theorem 2.2.

Case 1: $r+s \leq n-1$.

From (4.11) we know that $H_{\mathrm{BC}}^{r, s}\left(\mathcal{A}_{b}(M)\right)=\{0\}$ for $r+s \leq n-1$. Moreover, from Theorem 4.1 (the first isomorphism of (iii)) we have

$$
\begin{aligned}
\widetilde{H}_{b, \mathrm{BC}}^{r, s}(M) & \cong\left\{\varphi \in \mathcal{H}_{b, \mathrm{BC}}^{r, s}(M) \mid \varphi \wedge d \eta \in \partial_{b} \bar{\partial}_{b}\left(F_{b}^{r, s}(M)\right)\right\} \\
& \cong\left\{\varphi \in \mathcal{H}_{b, \mathrm{BC}}^{r, s}(M) \mid \varphi \wedge d \eta=0\right\} .
\end{aligned}
$$

Thus, from Lemma 3.2 we conclude that $\widetilde{H}_{b, \mathrm{BC}}^{r, s}(M)=\{0\}$ for $r+s \leq n-1$. This finishes the proof for $r+s \leq n-1$.

Case 2: $r+s \geq n+2$.

We will see that the mapping $\alpha_{r, s}$ given by (4.13) is an isomorphism for $r+s \geq$ $n+1$. By Proposition 4.5 it suffices to show the injection.

Let $a \in H_{\mathrm{BC}}^{r, s}\left(\mathcal{A}_{b}(M)\right)$ be such that $\alpha_{r, s}(a)=0$ in $\widetilde{H}_{b, \mathrm{BC}}^{r, s}(M)$ and suppose that $\varphi$ is a representative of $a$. Since $\alpha_{r, s}(a)=\alpha_{r, s}\left(\{\varphi\}_{\mathrm{BC}}\right)=[\varphi]_{\mathrm{BC}}=0$ in $\widetilde{H}_{b, \mathrm{BC}}^{r, s}(M)$, there exists $\psi \in F_{b}^{r-1, s-1}(M)$ such that

$$
\varphi=\partial_{b} \bar{\partial}_{b} \psi .
$$

Suppose that $\psi \notin \mathcal{A}_{b}^{r-1, s-1}(M)$ (notice that if $\psi \in \mathcal{A}_{b}^{r-1, s-1}(M)$, then $a=0$, and we conclude the proof). Since $L$ commutes with $\partial_{b}$ and $\bar{\partial}_{b}$, we have $\partial_{b} \bar{\partial}_{b}(L \psi)=$ $L\left(\partial_{b} \bar{\partial}_{b} \psi\right)=L \varphi=0$; therefore, $L \psi$ defines a basic Aeppli cohomology class $[L \psi]_{A} \in H_{b, A}^{r, s}(M)$. From Theorem 4.2 (the isomorphism (iii)) we have

$$
L \psi=\psi_{1}+\partial_{b} \gamma_{1}+\bar{\partial}_{b} \gamma_{2}
$$

for $\psi_{1} \in \mathcal{H}_{b, A}^{r, s}(M), \gamma_{1} \in F_{b}^{r-1, s}(M)$, and $\gamma_{2} \in F_{b}^{r, s-1}(M)$. Since $r+s \geq n+2$ and $\psi_{1} \in \mathcal{H}_{b, A}^{r, s}(M)=\mathcal{H}_{b}^{r, s}(M)$, by Lemma 3.3 there exists $\psi_{2} \in \mathcal{H}_{b}^{r-1, s-1}(M)=$ $\mathcal{H}_{b, A}^{r-1, s-1}(M)$ such that $L \psi_{2}=\psi_{1}$, and since $r+s-1 \geq n+1$, by Lemma 3.2 
there exist $\sigma_{1} \in F_{b}^{r-2, s-1}(M)$ and $\sigma_{2} \in F_{b}^{r-1, s-2}(M)$ such that $\gamma_{1}=L \sigma_{1}$ and $\gamma_{2}=L \sigma_{2}$, respectively. Thus,

$$
L\left(\psi-\psi_{2}-\partial_{b} \sigma_{1}-\bar{\partial}_{b} \sigma_{2}\right)=0 \quad \text { and } \quad \partial_{b} \bar{\partial}_{b}\left(\psi-\psi_{2}-\partial_{b} \sigma_{1}-\bar{\partial}_{b} \sigma_{2}\right)=\varphi .
$$

Then, $a=\{\varphi\}_{\mathrm{BC}}$ is the zero basic class in $H_{\mathrm{BC}}^{r, s}\left(\mathcal{A}_{b}(M)\right)$, and this finishes the proof.

Now, using the previous result, by similar arguments as in the proof of Theorem 3.2 we obtain a Hodge decomposition theorem for the coeffective basic BottChern cohomology of compact Sasakian manifolds.

THEOREM 4.4. If $M$ is a compact Sasakian manifold of dimension $2 n+1$, then we have:

(i) $\widetilde{H}^{p}(M) \cong \bigoplus_{r+s}^{p} \widetilde{H}_{b, \mathrm{BC}}^{r, s}(M)$;

(ii) $H^{p}\left(\mathcal{A}_{b}(M)\right) \cong \bigoplus_{r+s}^{p} H_{\mathrm{BC}}^{r, s}\left(\mathcal{A}_{b}(M)\right)$ for $r+s \geq n+2$.

Finally, let us denote by $c_{b, \mathrm{BC}}^{r, s}(M)=\operatorname{dim} H_{\mathrm{BC}}^{r, s}\left(\mathcal{A}_{b}(M)\right)$. Then we have the following:

Corollary 4.3. If $M$ is a compact Sasakian manifold of dimension $2 n+1$, then

$$
c_{b}^{p}(M)=\sum_{r+s=p} c_{b, \mathrm{BC}}^{r, s}(M), \quad \text { for } r+s \geq n+2 .
$$

\section{References}

[1] A. Aeppli, Some exact sequences in cohomology theory for Kähler manifolds, Pacific J. Math. 12 (1962), no. 3, 791-799.

[2] L. C. De Andrés, M. Fernández, M. de León, R. Ibáñez, and J. Mencía, On the coeffective cohomology of compact symplectic manifolds, C. R. Math. Acad. Sci. Paris 318 (1994), 231-236.

[3] D. E. Blair, Contact manifolds in Riemannian geometry, Lecture Notes in Math., 509, Springer, Berlin, 1976.

[4] , Riemannian geometry of contact and symplectic manifolds, Progr. Math., 203, Birkhäuser Boston, Inc., Boston, MA, 2002.

[5] B. Bigolin, Gruppi di Aeppli (Italian), Ann. Scuola Norm. Sup. Pisa (3) 23 (1969), 259-287.

[6] T. Bouché, La cohomologie coeffective d'une variété symplectique, Bull. Sci. Math. 114 (1990), no. 2, 115-122.

[7] C. P. Boyer and K. Galicki, Sasakian geometry, Oxford Math. Monogr., Oxford University Press, Oxford, 2008.

[8] D. Chinea, M. de León, and J. C. Marrero, Topology of cosymplectic manifolds, J. Math. Pures Appl. (9) 72 (1993), no. 6, 567-591.

[9] Coeffective cohomology on cosymplectic manifolds, Bull. Sci. Math. 119 (1995), no. 1, 3-20.

[10] D. Chinea, J. C. Marrero, and M. de Leon, A canonical differential complex for Jacobi manifolds, Michigan Math. J. 45 (1998), 547-579.

[11] A. El Kacimi Alaoui, Opérateurs transversalement elliptiques sur un feuilletage riemannien et applications, Compos. Math. 73 (1990), 57-106. 
[12] A. El Kacimi Alaoui and B. Gmira, Stabilité du caractère Kählérien transverse, Israel J. Math. 101 (1997), 323-347.

[13] A. El Kacimi Alaoui and G. Hector, Décomposition de Hodge basique pour un feuilletage riemannien, Ann. Inst. Fourier (Grenoble) 36 (1986), no. 3, 207-227.

[14] M. Fernández, R. Ibáñez, and M. de León, Coeffective and de Rham cohomologies of symplectic manifolds, J. Geom. Phys. 27 (1998), no. 3-4, 281-296.

[15] Coeffective and de Rham cohomologies on almost contact manifolds, Differential Geom. Appl. 8 (1998), 285-303.

[16] _ Coeffective cohomology of quaternionic Kähler manifolds, New developments in differential geometry, Budapest, 1996 (J. Szenthe, ed.), pp. 111-121, Springer Science+Business Media, Dordrecht, 1999.

[17] T. Fujitani, Complex-valued differential forms on normal contact Riemannian manifolds, Tôhoku Math. J. 18 (1966), 349-361.

[18] R. Ibáñez, Coeffective-Dolbeault cohomology of compact indefinite Kähler manifolds, Osaka J. Math. 34 (1997), 553-571.

[19] C. Ida and G. Pitiş, Basic cohomologies on K-contact manifolds, preprint, submitted.

[20] S. Kobayashi and K. Nomizu, Foundations of differential geometry, I, 1963, II, 1969, Interscience Publ., New York/London.

[21] J. Morrow and K. Kodaira, Complex manifolds, AMS Chelsea Publ., Providence, RI, 1971.

[22] Y. Ogawa, On C-harmonic forms in a compact Sasakian space, Tôhoku Math. J. 19 (1967), 267-296.

[23] G. Pitiş, Contact geometry: Sasaki manifolds, Kenmotsu manifolds, manuscript (unpublished).

[24] M. Schweitzer, Autour de la cohomologie de Bott-Chern, Prépublications de Inst. Fourier 703 (2007), www-fourier.ujf-grenoble.fr/prepublications.html, arXiv:0709.3528v1 [math].

[25] N. Tanaka, A differential geometric study on strongly pseudo-convex manifolds, Lectures in mathematics, 9, Kinokuniya, Tokyo, 1975.

[26] P. Tondeur, Foliations on Riemannian manifolds, Universitext, Springer-Verlag, New York, 1988.

[27] L.-S. Tseng and S.-T. Yau, Cohomology and Hodge theory on symplectic manifolds: I, II, J. Differential Geom. 91 (2012), no. 3, 383-416, 417-444.

[28] I. Vaisman, Cohomology and differential forms, M. Dekker Publ. House, New York, 1973.

C. Ida

Department of Mathematics and Computer Science

University Transilvania of Braşov

Braşov 500091

Str. Iuliu Maniu 50

România

\section{P. Popescu}

Department of Applied Mathematics

University of Craiova

Craiova, 200585

Str. Al. Cuza, No. 13

România 